
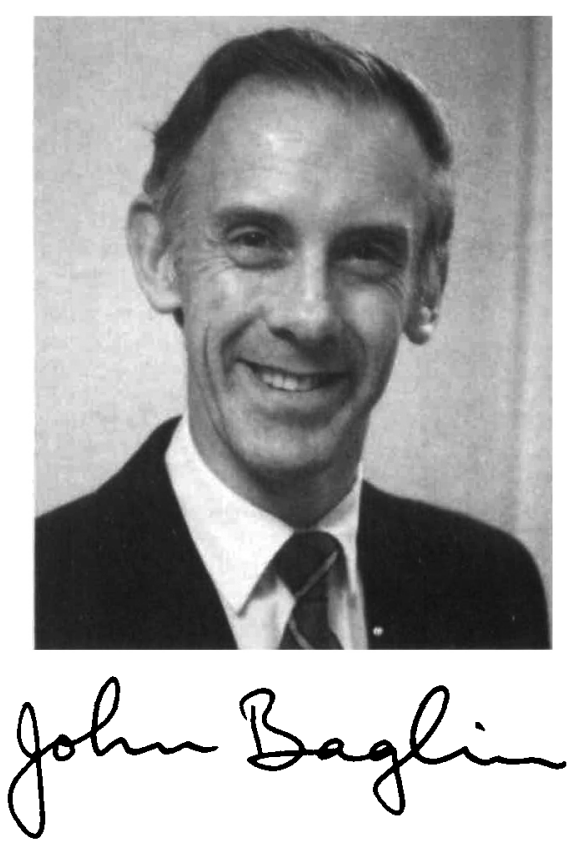

How are MRS symposium topics chosen for each meeting? We have a great idea for a future MRS symposium... Who should we talk to about it? Why isn't my topic scheduled at the next meeting?

Such questions have recently been asked of me with increasing frequency. On the one hand, I am very happy that so many people feel so much involvement with the Materials Research Society. However, on the other hand, I am concerned that perhaps we have not explained the process of creating a meeting program clearly enough that researchers with good proposals can make timely and effective contributions to the process. I would like to rectify that omission here, since your continued constructive participation is really appreciated, not only in meeting planning but in all aspects of MRS activity. We encourage your informed interest in the planning process, and we do welcome your unsolicited input.

\section{Proposals}

If you have a good idea for a future symposium, please write a title, a concise description of the topics to be included, and a couple of sentences saying why the topic is of current significance and why it is appropriate for an MRS meeting. Suggest potential organizers if you can. A form for this purpose is published occasionally in the MRS BULLETIN, but you need not stick to the form. Simply mail your proposal to the Chair of the Program Committee (Jim Roberto for 1988), with copies to Meeting Chairs if you have a particular meeting in mind.

In making your proposal, please remember that its chance of success will be en-

\title{
How are MRS Symposium Topics Chosen?
}

hanced if the topic is clearly specified and focused, with emphasis on areas of significant materials research activity and excitement (present or expected). Topics that will enrich our interdisciplinary activity are especially welcome, as are those in emerging areas of research.

Also important to the Materials Research Society is a growing number of topical research "communities" whose interests are a key part of MRS activities, and who have evolved patterns of organizing MRS symposia on a periodic basis. It is important that symposium proposals from these groups be made as soon as the group's intentions are known, using the guidelines I described above.

\section{Deadlines}

Formation of the ensemble of symposia for each meeting begins a long time in advance. For proper consideration by the Meeting Chairs and Program Committee, a proposal should be received at least one month prior to the third MRS meeting preceeding your target meeting. For example, proposals for symposia at the Spring 1990 meeting should be in hand by late October 1988. That does not mean that we cannot accommodate hot new topics should they develop. We maintain a degree of flexibility many other organizations envy-and whose reward is our ability to address an important subject at short notice, if need be. Our high- $T_{c}$ superconductor symposium in Spring 1987 was just such a case. However, in general, you need to marshal your ideas for a symposium about two years ahead of the event.

\section{Symposium Selection}

We believe that the highest quality meetings will continue to result when we invite the Meeting Chairs to draft the symposium list in their own way, limited by only a few key guidelines. Their broad goal is to make a meeting that is the most fruitful experience possible, and that best serves the materials research community. Symposia in related areas should be constructively integrated; developing research areas should be included; significant new topics should be explored.

The Program Committee assists the Meeting Chairs in this process in several ways-providing records of symposium proposals received and those carried forward from previous years, providing information on the recent history of repeating topics, listing dates of other technical society meetings that may present topical conflicts, and independently suggesting timely symposium topics for consideration.

The draft program developed by the Meeting Chairs is then refined in discussions with the Program Committee and the Executive Committee. After this, invitations to potential Symposium Organizers are made by the Meeting Chairs, who will select organizers representing an appropriate diversity of interests, special abilities, and affiliations. Subsequently, the Meeting Chairs set up a planning session with the new symposium organizers, and the Meeting is on its way to becoming a reality.

\section{Limited Capacity}

Our present guidelines limit us to about 20-25 symposia in any meeting, since holding more symposia could unacceptably lower the quality of the meeting due to conflicting schedules and overcrowded meeting space. Therefore, the Meeting Chairs must use a crystal ball and the wisdom of Solomon in order to select from all the candidate topics. With the expansion of the Materials Research Society in recent years, the pressure to repeat topics is being felt for an increasing number of symposia; however, we are also aware of intriguing new symposium topics where MRS can uniquely serve the community. With both Spring and Fall meetings constrained to 20 25 topics each, we find there is simply not the capacity to run every attractive symposium, as we could have done a few years ago.

Because of this situation, you can understand how very important it is that the Program Chair and Meeting Chairs receive your explicit proposals for future symposia in time to give them proper consideration. 
Please don't assume that your special topic, which ran previously, will automatically be scheduled again without your input. Although we hope we are aware of areas that should be regularly addressed (perhaps in fresh ways), we need to have explicit notice when enthusiasts in a particular area want to hold a follow-up symposium in a future year. This will ensure that good ideas and good intentions are not overlooked in the early planning stages.

\section{Future Plans}

Our growth and success have led us to a situation where we already have attractive symposium ideas we cannot accommodate in the present format of our meetings. I would be sad to see competition for future symposium slots rise to a point where our ability to serve the materials research community well would be compromised.

I believe we must now face squarely the options for dealing with this matter in the future. Should our meetings be allowed to expand to suit demand? Should we in- crease the number of meetings each year? Or should we simply filter and refine the program content of the existing meetings? Perhaps meetings of a different format should be tried? Members of the MRS Long-Range Planning Committee and Program Committee will have these questions in mind as long-term proposals are formulated this summer. Your opinions and constructive ideas would be welcomed by the chairs of both committees (Kathy Taylor and Jim Roberto). I should like to hear from you, too.

\section{The MRS Program Committee Needs Your Input \\ Suggestions for Future Symposia}

Symposium title:

Contact or suggested organizers:

Phone

Briefly describe the scientific background of the symposium:

What would be the technical content and purpose of the symposium?

When should this symposium run:

$$
\text { (Fall/Spring) }
$$

Are there any topical areas that you feel the Society should specifically address over the next few years? Please list:

Other suggestions for improving the technical program at our meetings; 